\title{
Retraso en el desarrollo del lenguaje oral y aprendizaje significativo en niños de 4 y 5 años
}

\section{Delayed oral language development and significant learning in 4- and 5-}

$$
\text { year-olds }
$$

1 María Gabriela Santana Tamayo $\quad$ (iD) https://orcid.org/0000-0002-3161-0069

Universidad Técnica de Ambato, Facultad de Ciencias Humanas y de la Educación,

Carrera de Psicopedagogía. Ambato, Ecuador.

msantana4442@uta.edu.ec

2 Alison Pamela Vayas Masache (iD) https://orcid.org/0000-0003-0406-8187

Universidad Técnica de Ambato, Facultad de Ciencias Humanas y de la Educación,

Carrera de Psicopedagogía. Ambato, Ecuador.

avayas0023@uta.edu.ec

3 Mishell Carolina Mancheno López (iD) https://orcid.org/0000-0001-7316-3175

Universidad Técnica de Ambato, Facultad de Ciencias Humanas y de la Educación,

Carrera de Psicopedagogía. Ambato, Ecuador.

mmanchen01634@uta.edu.ec

4 Tamara Yajaira Ballesteros Casco (iD) https://orcid.org/0000-0003-0406-818

Universidad Técnica de Ambato, Facultad de Ciencias Humanas y de la Educación,

Carrera de Educación Inicial. Ambato, Ecuador.

ty.ballesteros@uta.edu.ec

Artículo de Investigación Científica y Tecnológica

Enviado: 24/12/2021

Revisado: 29/12/2021

Aceptado: $12 / 01 / 2022$

Publicado:08/03/2023

DOI: https://doi.org/10.33262/concienciadigital.v6i1.4.2007

Cátese: Santana Tamayo, M. G., Vayas Masache, A. P., Mancheno López, M. C., \& Ballesteros Casco, T. Y. (2023). Retraso en el desarrollo del lenguaje oral y aprendizaje significativo en niños de 4 y 5 años. ConcienciaDigital, 6(1.4), 437-455. https://doi.org/10.33262/concienciadigital.v6i1.4.2007

CONCIENCIA DIGITAL, es una Revista Multidisciplinar, Trimestral, que se publicará en soporte electrónico tiene como misión contribuir a la formación de profesionales competentes con visión humanística y crítica que sean capaces de exponer sus resultados investigativos y científicos en la misma medida que se promueva mediante su intervención cambios positivos en la sociedad. https://concienciadigital.org _

La revista es editada por la Editorial Ciencia Digital (Editorial de prestigio registrada en la Cámara Ecuatoriana de Libro con No de Afiliación 663) www.celibro.org.ec 


\section{Palabras}

claves:

lenguaje oral, aprendizaje significativo, desarrollo, etapas, retraso del lenguaje.

Keywords: oral language, significant learning, developing, phase, language delay
Resumen

Introducción. El lenguaje es inherente a los seres humanos y constituye un elemento fundamental para relacionarnos con otras personas y comprender el mundo que nos rodea. Es por eso que la evaluación del lenguaje oral a edades tempranas es esencial para evitar retrasos e inclusive trastornos del lenguaje. En connotación a ello, el aprendizaje significativo puede verse afectado debido a un retraso en el lenguaje oral. Objetivo. Establecer la relación que existe entre el desarrollo del lenguaje oral y el aprendizaje significativo en niños de 4 a 5 años de edad. Metodología. Se aplica un enfoque mixto en 60 estudiantes de inicial II y primero de educación general básica de la Unidad Educativa "UK" modalidad semipresencial, mediante la aplicación de una lista de cotejo con indicadores respecto al Desarrollo del lenguaje oral como y el Aprendizaje Significativo. Resultados. Existe una clara correlación entre las dos variables, de este modo el desarrollo del lenguaje oral es uno de los principales factores que influye directamente en la adquisición de aprendizajes significativos. Conclusión. Existen pocos procesos a mejorar en el área del lenguaje oral para los niños, cabe recalcar que esto no afecta significativamente en su aprendizaje. Sin embargo, es primordial fortalecer dichas falencias con el propósito de lograr un óptimo y eficaz proceso de aprendizaje.

Abstract

Introduction. Language is inherent to human beings and constitutes a fundamental element for relating to other people and understanding the world around us. That is why the evaluation of oral language at an early age is essential to avoid delays and even language disorders. In connotation, meaningful learning can be impaired due to a delay in oral language. Objective. Establish the relationship between oral language development and meaningful learning in children 4 to 5 years of age. Methodology. It is applied a mixed approach is applied in 60 students of initial II and first of basic general education of the Educational Unit "UK" blended modality, through the application of a checklist with indicators regarding the Development of oral language as and Significant Learning Results. There is a clear correlation between the two variables, thus the development of oral language is one of the main factors that directly influences the acquisition of significant 
learning. Conclusion. There are few processes to improve in the area of oral language by children, it should be emphasized that this does not significantly affect their learning. However, it is essential to strengthen these shortcomings in order to achieve an optimal and effective learning process.

\section{Introducción}

A razón de que el ser humano es un ser social por excelencia, el lenguaje resulta imprescindible. Por tanto, González (2020) afirma que el lenguaje constituye la herramienta cognitiva que permite establecer una conexión con el mundo para exteriorizar todo aquello que acontece en nuestro pensamiento y también para explicar lo que sucede fuera. Cabe mencionar que el proceso de adquisición, evolución y optimización del lenguaje requiere de tiempo y práctica constante. Adicionalmente, el lenguaje tiene una vía impresiva, es decir la forma en que se recepta la información; la audición, la kinestesia y la compresión de las palabras por medio de los sentidos. Por otra parte, la vía expresiva se vincula con la manifestación, articulación y emulación de sonidos, palabras y frases con significado y sentido (Vigotsky, 2017).

\section{Tipos de lenguaje}

En relación con lo anteriormente expresado, Alonso (2018) señala que el lenguaje es un sistema de transmisión informativa por medio de distintos signos en relación con sonidos, gestos, letras, símbolos e imágenes. En dependencia con el signo empleado se dará el surgimiento de distintitos tipos de lenguaje, por ejemplo: lenguaje natural, lenguaje verbal, lenguaje no verbal, lenguaje corporal, lenguaje visual, lenguaje sonoro, lenguaje táctil, lenguaje olfativo, lenguaje matemático, lenguaje icónico, entre otros. Esto servirá para creación de mensajes según la necesidad, capacidad y contexto que serán interpretados por el mundo exterior (Manzano, 2018).

\section{Lenguaje oral}

Según Salvador (2017), el lenguaje oral se lo conoce como un acto comunicativo natural que se da por medio de la producción de sonidos verbales mediante al aparato fonador el cual es distinguido por el órgano del oído. A la vez sirve para la locución de pensamientos, sentimientos, sensaciones, interrelación y satisfacción de necesidades. Por ende, se lo interpreta como un proceso que está establecido en base a la expresión y la interpretación que permite que el acto comunicativo adquiera lógica y sentido.

De este modo, al hablar de desarrollo del lenguaje oral, hace referencia a las distintas etapas que intervienen en el proceso evolutivo del ser humano en ciertos periodos de 
tiempo, fundamentada en los diferentes procesos madurativos del sistema nervioso central en conjunto con el aprendizaje de la lengua natural materna, el entorno físico y social (Bonilla et al., 2020). Esto ayudará a que se desenvuelvan eventualmente las características básicas del lenguaje y de esta forma se concreten los procesos cognitivos, lo cual ayudará a la potencialización comunicativa que permitirá una interacción enriquecedora con el ambiente al cual está inmerso el sujeto (Melgar, 2017).

\section{Etapa prelingüística}

En adición, señalan que en la etapa sensoriomotora se desarrolla la etapa prelingüística, la cual se da desde los 0 hasta 12 meses, en la cual se empiezan a evidenciar reacciones ante sonidos del exterior, imitación de sonidos, afirmaciones por medio del asentamiento de la cabeza, señalamiento, entre otros, lo cual posibilita que se desarrollen habilidades y comportamientos que fomentan a la interacción con personas de su entorno (López et al., 2020).

\section{Etapa lingüística}

La etapa lingüística tiene su inicio al 1 año y se desarrolla hasta los 6 años con lo cual acontece la locución de la primera palabra emitida y esta se estima entre el periodo de 15 a 18 meses. Dando surgimiento al incremento de vocabulario, formación de frases con sentido, distinción de géneros, comienzo de la conjugación de verbos, dominación de la gramática. A partir de los 4 años se tornan más cuestionadores, manejan frases de hasta 5 palabras y articulan un mínimo de 1500 palabras (Castañeda, 2018).

\section{Aspectos funcionales del lenguaje Uso cognitivo}

Cuando se habla de las funciones con las que cumple el lenguaje, se hace alusión principalmente a su uso semántico o cognitivo. Esta función implica la comprensión de los significados de las palabras para lo cual se requiere atravesar previamente los procesos de input y output de la información del lenguaje que se obtiene del medio exterior. Así pues, los niños que presentan divergencia en la evolución del lenguaje hablado tienden a expresar sus ideas, pensamientos y sentimientos de forma desordenada, repetitiva e innecesaria. De este modo, su mensaje con frecuencia está lleno de pausas y trastabillaciones (Apodaca \& Zepeda, 2019).

\section{Uso pragmático}

Castañeda (2018) afirma que el lenguaje también cumple con una función pragmática, en otras palabras, es la intención comunicativa del lenguaje para establecer un puente de conexión con el mundo que nos rodea. Al presentarse un desfase en esta habilidad, los niños presentan dificultades para el entendimiento de preguntas, relatar historias, comprender el contexto de la narrativa, utilizar pronombres, hacer inferencias, iniciar, 
continuar o culminar conversaciones, entre otras características.

Factores que repercuten en el retraso del desarrollo del lenguaje

Cruz \& Pimentel (2018) indican que, esta habilidad se va desarrollando acorde al entorno en el que se desenvuelve el sujeto, esto quiere decir que existirán diferentes factores psicosociales que intervendrán en el proceso del lenguaje oral de un niño los mismos que pueden ser directos o indirectos. Principalmente se toma en cuenta el ámbito social, económico y cultural; la familia cumple un rol fundamental debido a que desde los primeros años de edad se debe ser claro con el lenguaje que utilizamos principalmente en casa, como se pronuncian las palabras, como son receptadas y posteriormente producidas por los infantes e incluso poder percatarnos y prevenir ciertas dificultades que se pueden presentar a lo largo del proceso de la comunicación.

\section{Factores sociales}

Siendo seres sociales es necesario tomar en cuenta el lenguaje como una herramienta esencial en la comunicación, por esta razón es necesario mencionar al aspecto cultural debido a que dentro del mismo se determina la interacción entre civilizaciones. En palabras de Ocampo \& Uribe (2019), esto quiere decir que al tener distintas costumbres y tradiciones la pronunciación o la evolución del lenguaje será diferente según las regiones en las que los niños se interrelacionen.

Usualmente se suele dejar pasar ciertos errores de pronunciación en los niños e incluso los adultos los imitan sin percatarse que la forma de hablar del infante no está acorde a su edad cronológica. Por esta razón es importante conocer ciertos aspectos que nos permitan prevenir un retraso del lenguaje que no ser tratado a tiempo podría llegar a convertirse en un trastorno de aprendizaje. Existen ciertas características que repercuten en el desarrollo del niño, evidenciando una alerta en relación con sus pares. Por tanto, el infante no comprenderá una instrucción o una explicación, le será difícil mantener una conversación debido a que no logra comprender que es lo que verdaderamente quiere (Universidad de Alcalá, 2021).

Cuando se menciona un trastorno del lenguaje o un retraso del mismo puede llegar a causar preocupación y angustia dentro del núcleo familiar. Por esta razón es necesario conocer distintas características que pueden ser clave en el desarrollo del niño y sus progenitores pueden llegar a pasar por alto; el infante suele tener problemas para comprender que es lo que le dicen las demás personas debido a que no captan el significado de las palabras. Otro aspecto a mencionar es que suelen presentar dificultades para expresar sus pensamientos ya que no organizan las palabras que desean utilizar, no ordenan ni dan sentido a su idea y están conscientes del mensaje quieren dar, pero no logran exteriorizarlo. 


\section{Factores familiares}

Es necesario que los padres de familia, los docentes y las autoridades de la unidad educativa cuenten con capacitaciones que les permita identificar estas dificultades. Principalmente los docentes serán los encargados de detectar e informar a quien corresponda para posteriormente continuar con el proceso debido. Además, Auza (2019) manifiesta que en los niños suele presentarse con frecuencia el contar historias o acontecimientos sin sentido como en el tiempo el no comprender el "ayer, hoy y mañana", en el espacio en el que ocurrió "aquí, allá, cerca o lejos". Suelen presentar confusiones que hasta cierto punto se tornan normales, pero al ser corregidas tampoco comprende o cambia su forma de expresarse se deberá tomar medidas de evaluación, continuar con una evaluación y finalmente el diagnóstico acertado (Haba, 2018).

\section{Factores orgánicos}

Los factores orgánicos hacen referencia a la predisposición genética que tiene el niño al nacer con respecto a presentar un retraso en el desarrollo del lenguaje (Aldrete, 2017). Estudios científicos develan que existen diferencias en la adquisición del lenguaje, siendo las niñas quienes destacan de mejor manera y mucho antes que los niños en la articulación de palabras, por ende, 1 de cada 20 niños son más proclives a desarrollar un retraso en el lenguaje. Dentro de los factores orgánicos se consideran también los déficits auditivos que imposibilitan total o parcialmente el escuchar y por la tanto producir el habla; la presencia de un traumatismo craneal; retardo mental; problemas de índole psicológica, específicamente a nivel emocional; y debido a que los mecanismos neurofisiológicos y psíquicos aún no han llegado a su momento de maduración cognitiva y en cuanto a estructuras (Rey, 2017).

\section{Retraso en el desarrollo del lenguaje oral}

Las características que hacen evidente la presencia de un retraso en el desarrollo del lenguaje en los infantes se encuentran asociadas a falencias en las habilidades cognitivas. Por lo cual, existen dificultades respecto a la capacidad receptiva, es decir en la comprensión y establecimiento de relaciones entre los sonidos, letras y palabras del lenguaje hablado. Por otra parte, existen repercusiones en la capacidad expresiva que se refiere a la facultad para articular el lenguaje y comunicarse, lo cual puede resultar afectado como consecuencia del retraso en el lenguaje oral. Asimismo, a nivel emocional las deficiencias están relacionadas con la falta de independencia, seguridad y gestión de sus emociones (Sala, 2020).

De forma concreta, las peculiaridades que se manifiestan en el desfase del desarrollo normal del lenguaje verbal son diversas. De este modo, Bonilla (2016) asevera que existe dificultad para comprender las relaciones espaciales, el niño no hace uso de las palabras 
para solicitar lo que quiere, le resulta complejo el articular palabras largas, resulta complicado comprender lo que el infante expresa, la memoria verbal a corto plazo es deficiente, sustituyen $\mathrm{u}$ omiten letras y palabras debido a fallas en la conciencia fonológica, entre otros (Huarte, 2017).

De acuerdo con Antenaza \& Pérez (2018), en caso de que el retraso en el lenguaje se presente alrededor de los 4 años y no sea identificado oportunamente, existe una gran probabilidad de que esto escale a un trastorno permanente. Por tanto, un diagnóstico a tiempo y un tratamiento adecuado constituirían un proceso clave para la prevención o disminución de los síntomas de dicho retraso en el lenguaje oral. En adición, al iniciar la escolarización el niño presentará dificultades en el aprendizaje que mermarán la adquisición de nuevos conocimientos y su óptimo desarrollo.

Es preciso manifestar que en el retraso del lenguaje oral existen niveles de gravedad, lo cuales se catalogan acorde a características en base a las etapas del desarrollo cronológico. Por ello Hartnett (2019), indica que los progenitores deben centrar su atención en cualquier alteración que puedan notar en sus hijos en contraste con otros niños

de la misma edad. Esto se evidenciará al evaluar con la ayuda de un especialista, que es lo que el infante puede expresar y cuál es el nivel de comprensión con el que cuenta al receptar el mensaje de otros.

Por un lado, los indicios de un retraso leve en el desarrollo del lenguaje se caracterizan por la presencia frecuente de errores en la articulación de palabras con similar sonido y a nivel pragmático no se visualizan dificultades. Sin embargo, su comprensión y expresión es menor, ya que omiten el uso de artículos y confunden o sustituyen las consonantes, a menudo las segundas consonantes de las palabras (Aguilera \& Busto, 2020).

Por otro parte García (2017) contribuye que las características del retraso moderado en la evolución del lenguaje en niños de entre 4 -5 años son: carencia en la organización del discurso, una deficiente conciencia fonológica, no distinguen diptongos, su vocabulario es muy reducido. En la producción de oraciones utilizan de 6 a 7 palabras sin conexión alguna entre las mismas o por lado hacen uso de frases cortas y repetitivas, conocidas como muletillas.

\section{Estimulación del lenguaje oral}

Al hablar de estimulación del lenguaje, se refiere al proceso que se da desde los primeros años de vida. Este proceso es sumamente relevante debido a que durante esta edad el cerebro cuenta con mayor plasticidad cerebral, es decir, es más flexible al aprendizaje y a la absorción de nuevos conocimientos. Por esta razón es fundamental que desde muy pequeño reciban afecto como caricias, miradas, gestos, palabras de aliento, besos, entre otros; que le permitan desarrollar todos sus sentidos. Otro aspecto importante es que los 
padres no son solo emisores, si no deben aprender a ser receptores de lo que sus hijos desean o solicitan, de esta forma se obtendrá una comunicación eficaz.

La comunicación oral está formada a base de la implicación de dos tipos de aprendizaje. El primer aprendizaje hace referencia al predominio instrumental de la lengua el cual incluye la fonología, morfología, semántica y sintáctica y por otro lado se incluye la compresión y expresión la cual está orientada al segundo tipo de aprendizaje. Es por ello que existen vías estratégicas para los distintos tipos de edades que ayudan al desarrollo y estimulación del lenguaje oral. En este apartado se tomará en consideración estrategias para 4 y 5 años. Estas pueden estar enfocada en la incitación de cortas conversaciones individuales con temas del interés del niño, favorecimiento de la locución abierta con relación a las actividades cotidianas, la no limitación de la libre expresión; que ayudara a promover un ambiente adecuado de aprendizaje en cuanto al lenguaje y comunicación de forma oral (Cerdas \& Murillo, 2017).

Asimismo, existen otro tipo de recursos didácticos como son los pictogramas que ayudan a fomentar el lenguaje oral, mediante la capacidad de abstracción, relación, creatividad y sobre todo mejorar la ampliación de su vocabulario y pronunciación. Ya que estos actúan como signos o representación de objetos reales de la vida cotidiana del sujeto (Chiliquinga \& Guachamín, 2019).

\section{Aprendizaje Significativo}

De acuerdo con Ausubel (citado por Latorre, 2017), el aprendizaje significativo hace alusión al puente que se genera entre el conocimiento adquirido previamente y los aprendizajes nuevos. De esta forma, se establecen significados a las cosas, a través de las funciones ejecutivas como: la memoria, el razonamiento, la resolución de problemas, toma de decisiones, organización, síntesis, inducción, deducción, flexibilidad cognitiva, entre otros. Adicionalmente, este tipo de aprendizaje tiene lugar cuando relacionamos conceptos, símbolos, sonidos, recuerdos, acontecimientos importantes, y de esta forma perdura la información a largo plazo en la memoria (Vásquez et al., 2018).

\section{Dimensiones}

Existen varias dimensiones que ayudan a que se desarrolle el aprendizaje significativo. En primer lugar, la motivación determina el nivel de predisposición con que cuenta el niño; la comprensión permite tener una idea clara para un mejor entendimiento; la funcionalidad hace referencia a la activación de las redes cognitivas para poner en práctica lo aprendido en cualquier ámbito en que se desenvuelve el sujeto; y finalmente la participación activa da paso a que el estudiante logre construir su propio aprendizaje mediante la reflexión y el cuestionamiento (Carranza, 2017). 


\section{Relación del Aprendizaje Significativo y el Lenguaje Oral}

En relación a lo mencionado anteriormente, se manifiesta que existe una conexión del aprendizaje significativo con el lenguaje oral, ya que en el rango de edad de 4 a 5 años la absorción de nuevos conocimientos es mucho más factible a través de ciertos recursos didácticos. Estos pueden ser canciones, películas, videos, audiolibros, flashcards, entre otros, y gracias a esto los infantes pueden ampliar su vocabulario, mejorar su pronunciación y desarrollar habilidades sociales (Ruiz, 2021).

Es así que Moreira (2017), señala que, los niños asimilan la información transformándola en conocimiento útil, la cual se almacenará de forma permanente gracias a la práctica constante y consciente. Por tanto, para que el aprendizaje llegue a ser significativo, se recomienda que el docente indague el nivel de conocimientos previos de los estudiantes antes de impartir una nueva temática. Por tal motivo, la información previa

con la que cuenta el estudiante servirá de cimiento y vínculo para posteriormente reforzar y generar los aprendizajes esenciales (Ríos et al., 2021).

\section{Metodología}

El presente estudio tiene un enfoque mixto, es decir cuantitativo y cualitativo, es de tipo exploratoria-descriptiva, en un nivel de investigación correlacional y la modalidad es bibliográfica y de campo. Para la recolección de información se aplicó una lista de cotejo con 20 indicadores correspondientes al Desarrollo del lenguaje oral como primera variable y el Aprendizaje Significativo como segunda variable para ayudar detectar el retraso en el desarrollo del lenguaje. Dicha lista de cotejo fue aplicada a los padres de familia de los niños entre 4 y 5 años de inicial II y primero de educación general básica de la Unidad Educativa "UK" modalidad semipresencial, perteneciente a la provincia de Tungurahua, ciudad de Ambato.

\section{Resultados:}

\section{Tabla 1}

Condiciones

\begin{tabular}{lc}
\hline Escala ordinal & Equivalencia numérica \\
\hline Muy bien & 4 \\
Bien & 3 \\
Regular & 2 \\
Necesita mejorar & 1 \\
\hline
\end{tabular}

Fuente: Estudio de contexto UniKids 


\section{Figura 1}

Desarrollo del lenguaje oral - Repertorio de palabras

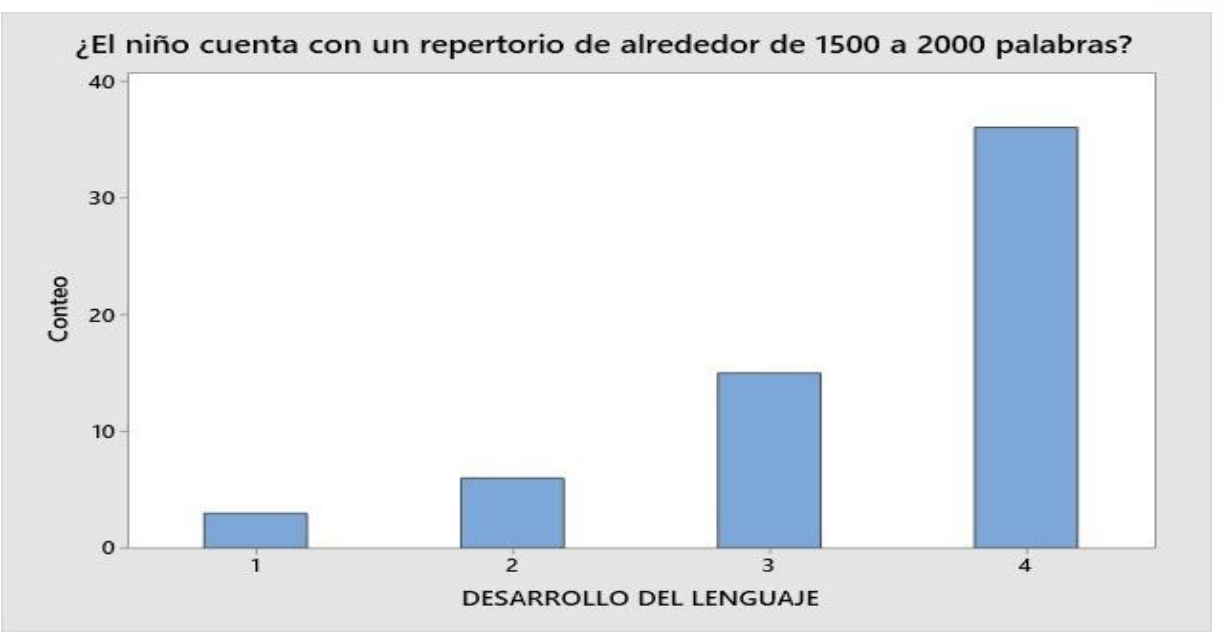

Nota: rango del repertorio de palabras con que cuentan los niños entre 4 y 5 años

Fuente: Estudio de contexto UniKids - Equipo de investigación

Tabla 2

Repertorio de palabras

\begin{tabular}{|c|c|c|c|c|c|c|c|}
\hline PREGUNTA & $\begin{array}{l}\text { MUY BIEN } \\
\%\end{array}$ & & BIEN\% & $\mathrm{REC}$ & $\mathrm{AR} \%$ & \multicolumn{2}{|c|}{$\begin{array}{l}\text { NECESITA } \\
\text { MEJORAR }\end{array}$} \\
\hline $\begin{array}{l}\text { ¿El niño cuenta con un repertorio de } \\
\text { alrededor de } 1500 \text { a } 2000 \text { palabras? }\end{array}$ & e $36 \quad 60 \%$ & 15 & $25 \%$ & 6 & $10 \%$ & 3 & $5 \%$ \\
\hline
\end{tabular}

Fuente: Estudio de contexto UniKids - Equipo de investigación

Del total de padres encuestados, 3 de ellos que equivale al 5\% consideran que sus hijos necesitan mejorar para ampliar su vocabulario y así cumplir con el desarrollo adecuado del lenguaje oral acorde a su edad, por otra parte 6 de ellos que corresponde al $10 \%$ manifiestan que sus hijos tienen un repertorio regular respecto a este indicador, 15 de los encuestados que es parte del $25 \%$ identifican que existe un buen manejo del repertorio de palabras por parte sus hijos, y 36 sujetos que constituye el $60 \%$ señalan que sus niños tienen un amplio vocabulario catalogándolo como muy bueno. En conjunto el número de encuestados suman 60 el mismo que representa al 100\% de la población de estudio. 


\section{Figura 2}

Desarrollo del lenguaje oral - Expresión oral inteligible

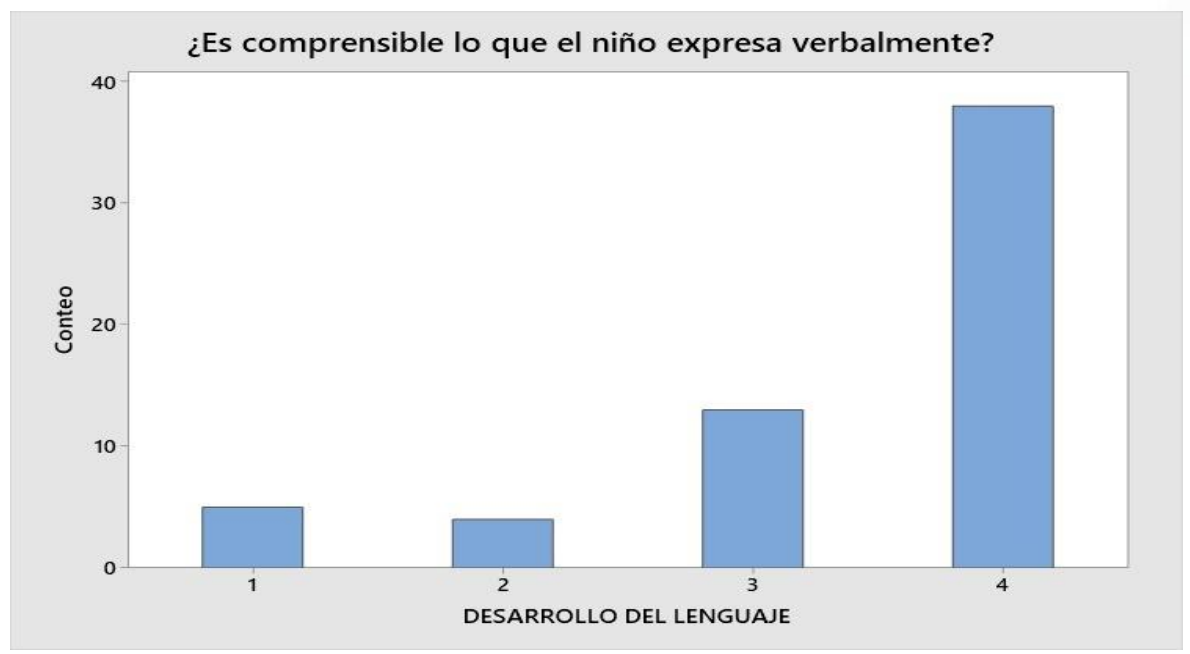

Nota: estándar de comprensión de la expresión oral del infante

Fuente: Estudio de contexto UniKids - Equipo de investigación

Tabla 3

Expresión oral inteligible

\begin{tabular}{|c|c|c|c|c|c|c|}
\hline PREGUNTA & MUY BIEN \% & & & $\mathrm{RE}$ & $\mathrm{LAR} \%$ & $\begin{array}{l}\text { NECESITA } \\
\text { MEJORAR }\end{array}$ \\
\hline $\begin{array}{l}\text { ¿Es comprensible lo que el niño } \\
\text { expresa verbalmente? }\end{array}$ & $3863.3 \%$ & 13 & $21 \%$ & 4 & $6.6 \%$ & $\begin{array}{ll}5 & \\
\% & 8.3\end{array}$ \\
\hline
\end{tabular}

Fuente: Estudio de contexto UniKids - Equipo de investigación

El total de encuestados son 60 participantes que representan al 100\%, 5 de los progenitores que conforman el $8.3 \%$ exponen que no se da una expresión verbal entendible por parte de sus hijos por lo que necesitan mejorar, por otro lado $6.6 \%$ de los encuestados, es decir 4 personas manifiestan que sus hijos tienen un lenguaje oral regular, en contraste a ello 13 de los padres que constituye el $21.7 \%$ indican que el habla de sus niños es buena, y 38 participantes que conforman el $63.3 \%$ consideran que sus hijos no presentan ningún problema en cuanto a la expresión oral, es decir es totalmente entendible. 


\section{Figura 3}

\section{Utilización de conocimientos adquiridos-Aprendizaje Significativo}

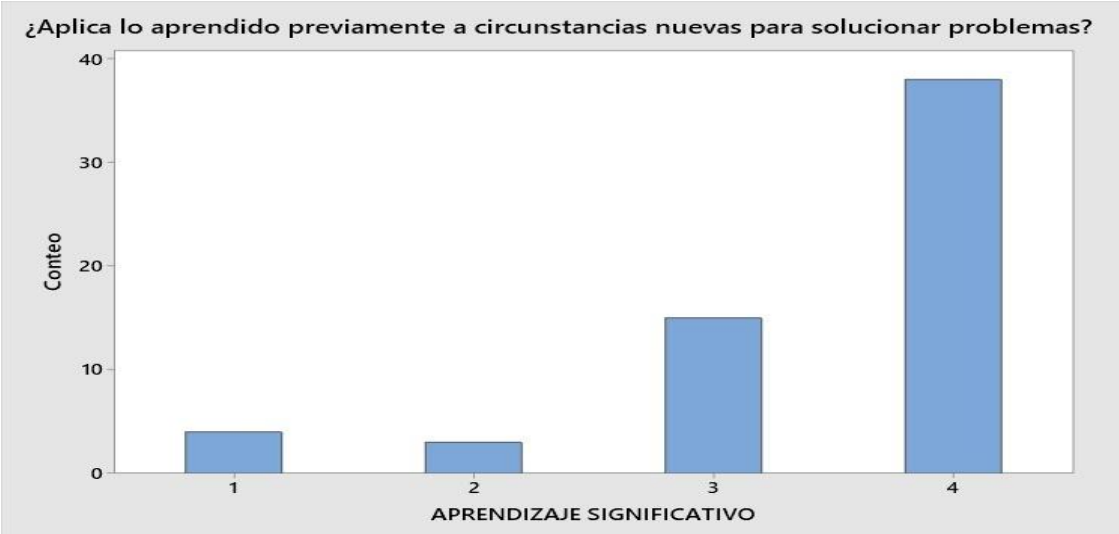

Nota: nivel de relación entre lo aprendido con anterioridad y los nuevos aprendizajes.

Fuente: Estudio de contexto UniKids - Equipo de investigación

Tabla 4

Utilización de conocimientos adquiridos

\begin{tabular}{|c|c|c|c|c|c|c|c|}
\hline \multirow{2}{*}{$\begin{array}{l}\text { PREGUNTA } \\
\text { ¿El niño }\end{array}$} & MUY BIEN \% & \multicolumn{2}{|c|}{ BIEN\% } & \multicolumn{2}{|c|}{ REGULAR\% } & \multicolumn{2}{|c|}{$\begin{array}{l}\text { NECESITA } \\
\text { MEJORAR }\end{array}$} \\
\hline & lo $\quad$ aprendido 38 & 15 & $25 \%$ & 5 & $8.3 \%$ & 4 & $6.6 \%$ \\
\hline
\end{tabular}

Fuente: Estudio de contexto UniKids - Equipo de investigación

Posterior a la evaluación, los representantes indicaron que 4 de los niños que son parte del $6.6 \%$ no aplica lo aprendido con anterioridad frente a problemas que se le presentan, por otra parte $8,3 \%$ de los mismos equivalente a 5 encuestados lo hacen de forma regular, mientras que otros 15 niños que conforman el $25 \%$ resuelven los problemas de forma adecuada, finalmente y en su mayoría con el $63.3 \%$ correspondiente a 38 padres de familia consideran que sus hijos solucionan muy bien los nuevos problemas, tomando en cuenta los aprendizajes previos. En total se obtuvieron 60 respuestas las mismas que constituyen al $100 \%$ de evaluados. 


\section{Figura 4}

\section{Deducción en base a diferentes contextos - Aprendizaje Significativo}

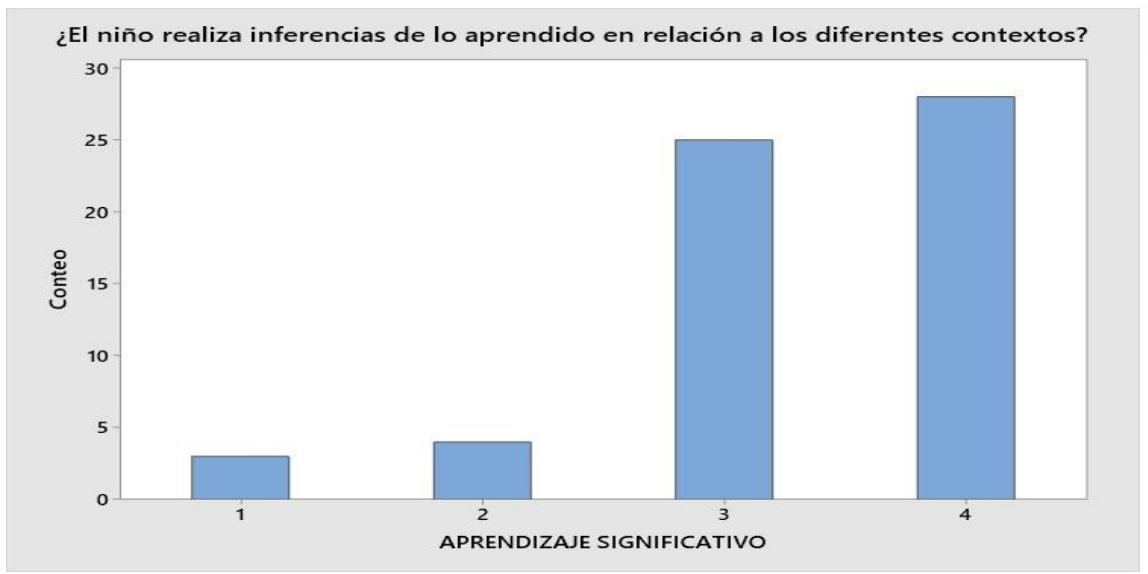

Nota: nivel de deducción de los aprendizajes en distintos contextos.

Fuente: Estudio de contexto UniKids - Equipo de investigación

\section{Tabla 5}

Deducción en base a diferentes contextos

\begin{tabular}{|c|c|c|c|c|c|c|}
\hline PREGUNTA & MUY BIEN \% & BIEN\% & & LAR\% & $\begin{array}{l}\text { NEC } \\
\text { MEJ }\end{array}$ & $\begin{array}{l}\text { ITA } \\
\text { RAR }\end{array}$ \\
\hline $\begin{array}{l}\text { ¿El niño realiza inferencias de lo } \\
46.6 \% \text { aprendido en relación a lo }\end{array}$ & entes & ${ }_{\%}^{25} 41.6$ & 4 & $6.6 \%$ & 3 & $5 \%$ \\
\hline
\end{tabular}

Fuente: Estudio de contexto UniKids - Equipo de investigación

De la sumatoria total los 60 participantes que corresponden al 100\% de evaluados, se puede observar que el 5\%, es decir 3 de ellos necesitan mejorar debido a que tienen ciertas dificultades para realizar inferencias respecto a lo que aprenden y relacionan con su contexto, $6.6 \%$ que equivale a 4 encuestados cumplen con dicho proceso de forma regular, mientras que 25 participantes que representan al $41.6 \%$ niños ejecutan bien este proceso, por último, el $46.6 \%$ que corresponde a los 28 padres de familia indican que sus hijos no tienen ningún problema al comprender que su aprendizaje puede adaptarse a distintos contextos iguales o diferentes al de ellos. 


\section{Figura 5}

Correlación de variables

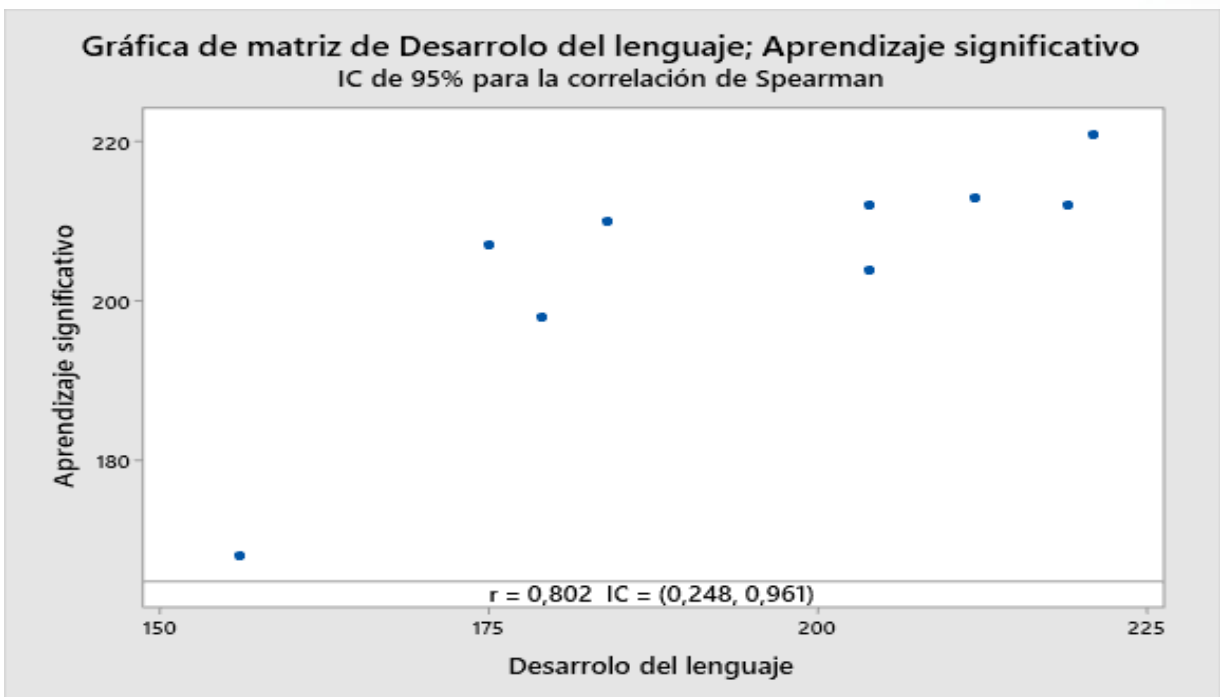

Fuente: Estudio de contexto UniKids - Equipo de investigación

La correlación existente entre el desarrollo de lenguaje oral y el aprendizaje significativo es de 0,802 , por ende, se infiere que los niños de entre 4 y 5 años de edad de la Unidad Educativa "UniKids" cuentan con un adecuado desenvolvimiento en cuanto a la expresión oral acorde a la etapa de desarrollo, vocabulario amplio, capacidad de relacionar y explicar de forma espontánea situaciones de la vida diaria y a la vez sus sentimientos, pensamientos y emociones. Así pues, existe la adquisición de aprendizajes útiles, duraderos, significativo y trascendentales para que su proceso educativo integral.

\section{Conclusiones}

- El Lenguaje oral entendido como un proceso cognitivo y acto comunicativo natural que permite a los seres humanos establecer una conexión con el mundo, se determina que mientras más temprano se favorece el desarrollo del lenguaje oral en los niños se establece cimientos seguros para los procesos adaptativos.

- Al encontrar dificultades en el desarrollo del lenguaje oral en la primera infancia, se precisa una intervención temprana tanto en el hogar como en las instituciones educativas con la finalidad de contrarrestar repercusiones sociales, escolares e individuales; a sapiencia de que en los primeros años del infante goza del privilegio de la plasticidad cerebral.

- La conexión del aprendizaje significativo con el lenguaje oral en la primera infancia es muy evidente debido a que los niños con el conocimiento previo del 
lenguaje, está en la capacidad de adquirir constantemente vocabulario nuevo y la comprensión de cómo funciona su aparato fonador para reproducir los sonidos de las palabras.

- Finalmente se hace hincapié en la importancia de buscar recursos que fortalezcan el desarrollo del lenguaje oral en niños en sus primeros años, estableciendo bases sólidas en su formación psicológica, anatómica e intelectual, que se refleje al momento de mantener conversaciones sencillas, de expresar sus pensamientos, emociones y disgustos con respeto e independencia; creando de esta manera seres humanos con conciencia del mundo que los rodea y del contexto en el que se desenvuelve; generando de esta manera verdaderos aprendizajes significativos perdurables en el tiempo.

\section{Referencias Bibliográficas}

Cerdas, J., \& Murillo, M. (2017). El desarrollo del lenguaje en los primeros cuatro años de vida, Revista Electrónica: Leer, Escribir y Descubrir, 31.

Chiliquinga, G., \& Guachamín, E. (2019). Los pictogramas en el desarrollo del lenguaje oral en niños y niñas de 3 - 4 años. Quito-Ecuador.

Aguilera, S., \& Busto, O. (2020). Retrasos y trastornos en el desarrollo del lenguaje. Pediatría Integral.

Aldrete, K. (2017). Factores que influyen en la adquisición de lenguaje. Barcelona.

Alonso, Á. (2018). ¿Qué es el lenguaje? Revista de Libros, 1-3.

Antenaza, Y., \& Pérez, L. (2018). Nivel de desarrollo del lenguaje oral en niños de 4 años de educación inicial de una Institución Educativa de Chiclayo. Chiclayo.

Apodaca, J., \& Zepeda, D. (2019). Desarrollo del aspecto semántico del lenguaje. CONISEN, 2-4.

Auza, A. (2019). Factores individuales y familiares en el Trastorno del Desarrollo del Lenguaje (TDL). Iztapalapa. Revista de ciencias sociales y humanidades.

Bonilla, P., Troya, E., \& Peñafiel, V. (2020). La utilización de la tecnología en el desarrollo del lenguaje de los niños de 4 años. Ciencia Digital, 63-79.

Bonilla, R. (2016). El desarrollo del lenguaje oral en niños de 4 años del colegio Hans. Repositorio Institucional, 66-67.

Carranza, M. (2017). Enseñanza y aprendizaje significativo en una modalidad mixta: 
percepciones de docentes y estudiantes. RIDE. Revista Iberoamericana para la Investigación y el Desarrollo Educativo.

Castañeda, P. F. (2018). Desarrollo del lenguaje verbal en el niño. Lima, Perú.

Cruz, L., \& Pimentel, S. (2018). Factores externos y el desarrollo del lenguaje en los preescolares en la institución educativa inicial San Judas Tadeo del distrito de breña, mayo- julio 2017.

García, V. (2017). Niveles de Gravedad en El Retraso Del Lenguaje. Guatemala. Gómez,

González, B. (2020). ¿Qué es el lenguaje? México: Acervo Digital Educativo.

Haba, B. I. (2018). Detección temprana de los trastornos específicos del neurodesarrollo del lenguaje mediante una prueba de repetición de palabras y pseudopalabras. Madrid.

Hartnett, J. (noviembre de 2019). Kids Health. https://kidshealth.org/es/parents/nottalk.html

Huarte, A. (2017). El retraso en el lenguaje. GAES Médica.

Latorre, M. (2017). Aprendizaje Significativo y Funcional.

López, M., Chamba, S., Zapata, A., \& Robles, G. (2020). Desde la perspectiva Neuropsicológica: la lateralidad, fluidez verbal, y conciencia fonológica componentes fundamentales en el desarrollo del proceso lecto escritor. Ciencia Digital, 24-41.

Manzano, P. (2018). La comunicación. Lenguaje verbal y lengua. El estudio de la lengua española. Madrid, España: Cerasa.

Melgar, S. R. (2017). Lenguaje oral y conciencia fonológica. Estudio empírico en una muestra de preescolares del distrito de Ventanilla-Callao. Eduser, 99-101.

Moreira, M. (2017). Aprendizaje significativo como un referente para la organización de la enseñanza.

Ocampo M, M., \& Uribe Á, G. (2019). www.researchgate.net. https://www.researchgate.net/profile/Hernan-

Obando/publication/343376888_Conceptualizacion_y_estrategias_mitigadoras_ de_la_violencia_de_genero_Una_mirada_desde_imaginarios_patriarcales_en_la _escuela_At_Revolucion_en_la_Formacion_2_Vol_I/links/5f25fec39285

Rey, M. (2017). Factores que influyen en el desarrollo el lenguaje: un estudio empírico 
en una muestra de niños y niñas de entre 3 y 4 años. Valladolid.

Ríos, R., Coral, S., Carrasco, O., \& Espinoza, C. (2021). La estimulación temprana como base para los procesos de enseñanza-aprendizaje en la educación infantil. Ciencia Digital, 252-271.

Ruiz, E. (2021). Fortalecimiento del aprendizaje significativo del Lenguaje. Quevedo.

Sala, M. (2020). Trastornos del lenguaje oral y escrito. Congreso de Actualización Pediatría 2020. Madrid: Lúa Ediciones 3.0.

Salvador, M. B. (2017). La importancia del lenguaje oral en educación infantil. Aula de Innovación.

Universidad de Alcalá. (2021). Detección e intervención educativa en dificultades de expresión y comunicación. Alcalá.

Vásquez, B., Espinoza, J., Hidalgo, W., Pasochoa, T., \& Jácome, D. (2018). Incidencia del aprendizaje significativo con el uso de B-Learning. Ciencia Digital, 366-381

Vigotsky, L. (2017). La teoría de Piaget sobre el aprendizaje. En Pensamiento y lenguaje (Tercera edición ed., págs. 6-7). Pueblo y Educación.

\section{\Cigital}


El artículo que se publica es de exclusiva responsabilidad de los autores y no necesariamente reflejan el pensamiento de la Revista Conciencia Digital.

\section{Ciencia \\ LDigital}

El artículo queda en propiedad de la revista y, por tanto, su publicación parcial y/o total en otro medio tiene que ser autorizado por el director de la Revista Conciencia Digital.
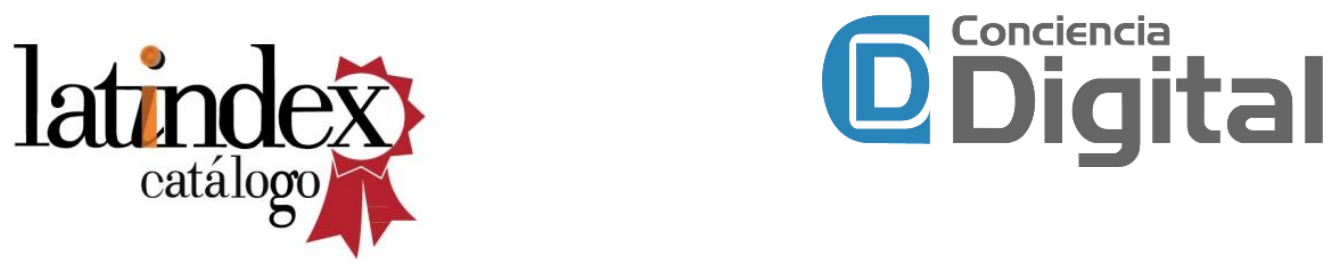

Indexaciones

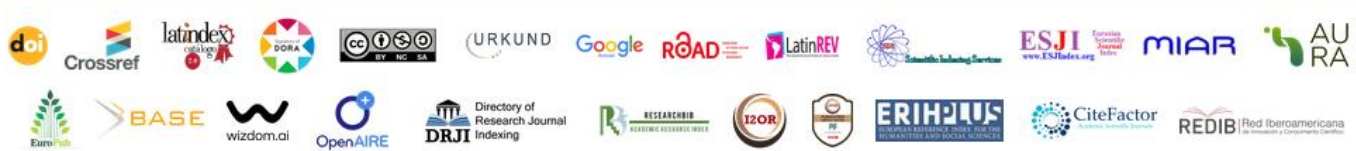

